\title{
Correction to: Artificial intelligence for diabetic retinopathy screening: a review
}

\author{
Andrzej Grzybowski • Piotr Brona • Gilbert Lim (D) Paisan Ruamviboonsuk • Gavin S. W. Tan • Michael Abramoff • \\ Daniel S. W. Ting (D)
}

Published online: 10 December 2019

(c) The Author(s), under exclusive licence to The Royal College of Ophthalmologists 2019

\section{Correction to: Eye}

https://doi.org/10.1038/s41433-019-0566-0

On Page 5, under the heading of Retinalyze, the following statement was made:

"A large report based on disease/no disease grading of 6722 patients was published in 2007 showing $90.5 \%$ sensitivity for detecting any DR or technical issues, with $67.4 \%$ specificity [35]."
This report has been erroneously placed under the Retinalyze subheading and does not represent results obtained using the Retinalyze system.

This has been removed from the PDF and HTML of the manuscript.

The authors sincerely apologise for this error. 